\title{
Immunofluorescent Staining Method
}

National Cancer Institute

\section{Source}

National Cancer Institute. Immunofluorescent Staining Method. NCI Thesaurus. Code C142337.

A microscopy staining method that utilizes immunofluorescent markers for use with histological applications of preserved cells. 